\title{
ON FINITE DIFFERENCES ON A STRING PROBLEM
}

\author{
${ }^{1}$ Mango, J.M., ${ }^{2}$ C. Eryenyu and ${ }^{3}$ S.E. Rugeihyamu \\ ${ }^{1}$ Department of Mathematics, Makerere University, Kampala, Uganda \\ ${ }^{2}$ Faculty of Science and Education, Busitema University, Busia, Uganda \\ ${ }^{3}$ Department of Mathematics, University of Dar-es-Salaam, Tanzania
}

Received 2013-04-12; Revised 2013-07-16; Accepted 2013-07-19

\begin{abstract}
This study presents an analysis of a one-Dimensional (1D) time dependent wave equation from a vibrating guitar string. We consider the transverse displacement of a plucked guitar string and the subsequent vibration motion. Guitars are known for production of great sound in form of music. An ordinary string stretched between two points and then plucked does not produce quality sound like a guitar string. A guitar string produces loud and unique sound which can be organized by the player to produce music. Where is the origin of guitar sound? Can the contribution of each part of the guitar to quality sound be accounted for, by mathematically obtaining the numerical solution to wave equation describing the vibration of the guitar string? In the present sturdy, we have solved the wave equation for a vibrating string using the finite different method and analyzed the wave forms for different values of the string variables. The results show that the amplitude (pitch or quality) of the guitar wave (sound) vary greatly with tension in the string, length of the string, linear density of the string and also on the material of the sound board. The approximate solution is representative; if the step width; $\partial \mathrm{x}$ and $\partial \mathrm{t}$ are small, that is $<0.5$.
\end{abstract}

Keywords: Guitar String, Finite Differences

\section{INTRODUCTION}

Ron (2002) defines a wave as, "A distortion in a material or medium where the individual parts of the material only cycle back-and-forth or up-and-down, but the wave itself moves through the material". Waves exist widely in nature, such as electromagnetic waves and in many other forms of mechanical waves. Waves carry energy. The energy carried by a wave is evident in many ways. Seismic waves carry enormous amount of energy that shake the earth resulting into earth quakes. Electromagnetic waves carry information in many forms enabling the use of communication through the internet, satellites, optical fibers and radios. The energy from microwaves is converted into thermal energy in microwave ovens. This study presents a mathematical analysis of mechanical waves from a guitar string. Alan (2000) noted that, "electromagnetic, microwaves between the earth and communication satellites, light waves in optical fibers are some of the ways in which wave energy is utilized". We can add that music is another way of utilizing wave energy.
The string is a material made of several threads twisted together. In this study, a string means a wire, a thread, nylon or any other synthetic material that is thin compared to its length, stretchable between two points. Therefore, a guitar string is any such material when used in a guitar.

A vibrating string is just a model of many objects that vibrates in nature. Most vibrations result into wave motion. As already noted waves carry energy that can be controlled for human benefits. The study of waves helps to model many things that are useful in everyday life. Musical instruments, engineering devices for weather forecast, tsunami and earthquake detection devices as well as basic communication devices. A vibrating string presents a better and an initial point to the study of waves because the variables can easily be manipulated. The vibration of a string is time and space dependent, a typical example of multidimensional systems. Lutz and Rudolf (2000) noted that "Multidimensional physical phenomena depending on time and space are commonly described by Partial Differential Equations (PDEs). Technical application of PDEs include electro-magnetic, optics, acoustics, heat and

Corresponding Author: Mango, J.M., Department of Mathematics, Makerere University, Kampala, Uganda 
mass transfer". We study a classical guitar string for its application in acoustics and entertainment.

\subsection{The Guitar}

A basic classical guitar consists of inter connected parts, these include: The head-containing the tuning pegs and head nut for alignment. The neck-this has the strings which are plucked or strummed to vibrate and produce sound, finger board and frets. The body-this consists of the soundboard, bridge and bridge nut (Fig. 1a).

John (1999) says, "99\% of the guitar sound comes from the soundboard". However, we have to note at this point that the soundboard is 'forced' to vibrate by the string and it vibrates at the same frequency as the string. In a guitar, the mechanical energy used in plucking the string is transferred into mechanical energy of vibration of the string. The string forces the sound board to vibrate which in turn transmit the vibration to the surrounding air. The vibration of the air creates regions of low and high pressure. These are detected by the human ear as sound (Fig. 1b).

\subsection{Wave Equation for a Vibrating Guitar String}

In the present research, the word 'string' is not used in the sence of 'string matching' algorithm as an essential means for searching biological sequence database as was used by Almazroi (2011), Al-mazroi and Rashid (2011).

Consider a single guitar string plucked from the center and $\mathrm{u}(\mathrm{x}, \mathrm{t})$ is the function describing a point along the string (Fig. 2a-b).

$T_{1}$ and $T_{2}$ are tangential tensions at points $x$ and $\mathrm{x}+\partial \mathrm{x}$ respectively. Let $\rho$ be mass per unit length of the string (linear density). The linear density is constant throughout the string, Alan (2000). As the wave propagates from point $\mathrm{x}$ to $\mathrm{x}+\partial \mathrm{x}$, the horizontal component of the tension remains a constant $\mathrm{T}$.

This means:
$\mathrm{T}_{1} \cos \theta=\mathrm{T}_{2} \cos \phi=\mathrm{T}$

Applying Newton's second law to vertical motion of the string, that is;

Resultant Force $=$ mass $\times$ acceleration, implies that:

$\mathrm{T}_{2} \sin \phi=\mathrm{T}_{1} \sin \theta=\rho \partial \mathrm{x} \frac{\partial^{2} \mathrm{u}}{\partial \mathrm{t}^{2}}$.

From Equation (1.1):

$$
\mathrm{T}_{1}=\frac{\mathrm{T}}{\cos \theta} \operatorname{andT}_{2}=\frac{\mathrm{T}}{\cos \phi} .
$$

Substituting for $\mathrm{T}_{1}$ and $\mathrm{T}_{2}$ in Equation (1.2) gives:

$\mathrm{T} \tan \phi-\mathrm{T} \tan \theta=\rho \partial \mathrm{x} \frac{\partial^{2} \mathrm{u}}{\partial \mathrm{t}^{2}}$.

Using elementary calculus:

$$
\left(\frac{\delta \mathrm{u}}{\delta \mathrm{x}}\right)_{\mathrm{x}}=\tan \theta \operatorname{and} \tan \left(\frac{\delta \mathrm{u}}{\delta \mathrm{x}}\right)_{\mathrm{x}+\partial \mathrm{x}}=\tan \phi
$$

Substituting in Equation (1.3) gives:

$$
\mathrm{T}\left[\left(\frac{\delta \mathrm{u}}{\delta \mathrm{x}}\right)_{\mathrm{x}+\partial \mathrm{x}}-\left(\frac{\delta \mathrm{u}}{\delta \mathrm{x}}\right)_{\mathrm{x}}\right]=\rho \partial \mathrm{x} \frac{\partial^{2} \mathrm{u}}{\partial \mathrm{t}^{2}}
$$

Equation (1.4) can be written as Equation (1.5):

$$
\frac{1}{\partial x}\left[\left(\frac{\delta u}{\delta x}\right)_{x+\partial x}-\left(\frac{\delta u}{\delta x}\right)_{x}\right]=\frac{\rho^{2} \partial u}{T \partial t^{2}} .
$$

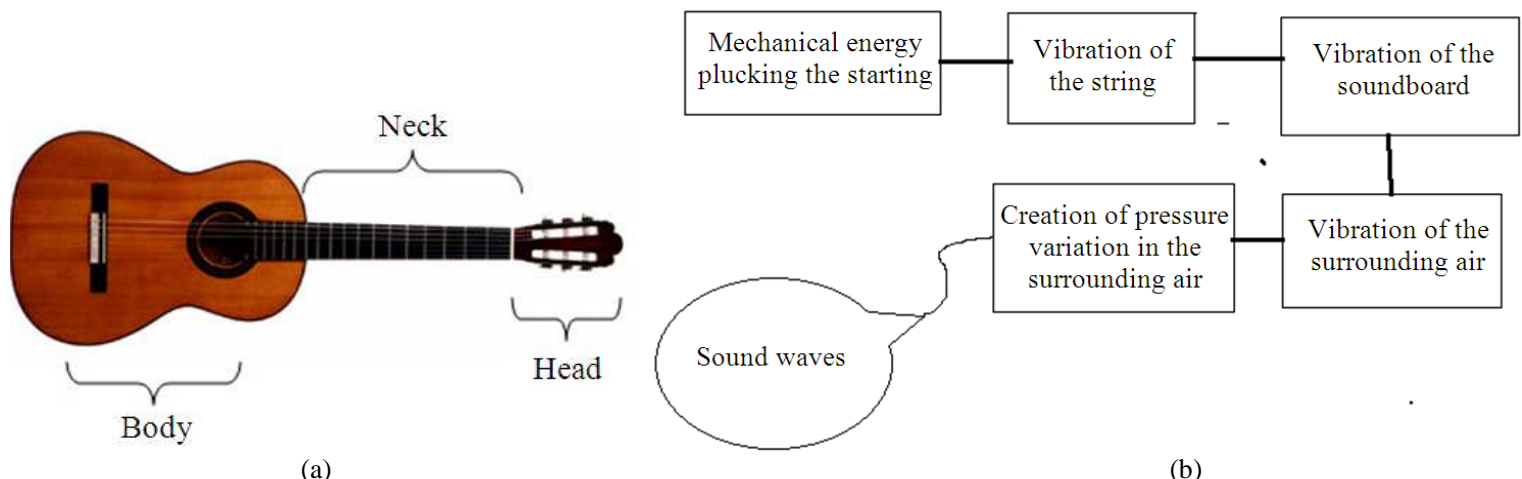

(a)

(b)

Fig. 1. (a) Parts of a guitar (b) Energy transfer in a guitar 


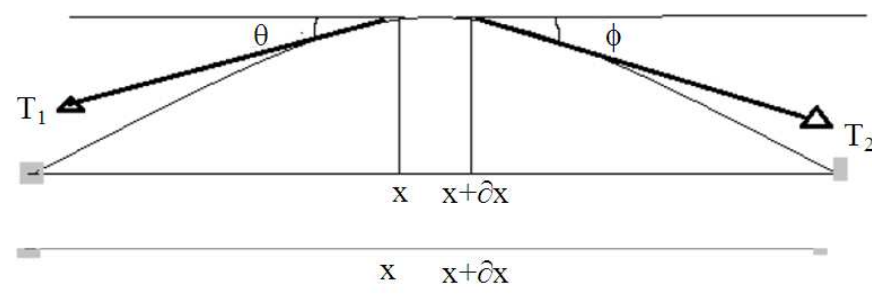

(a)

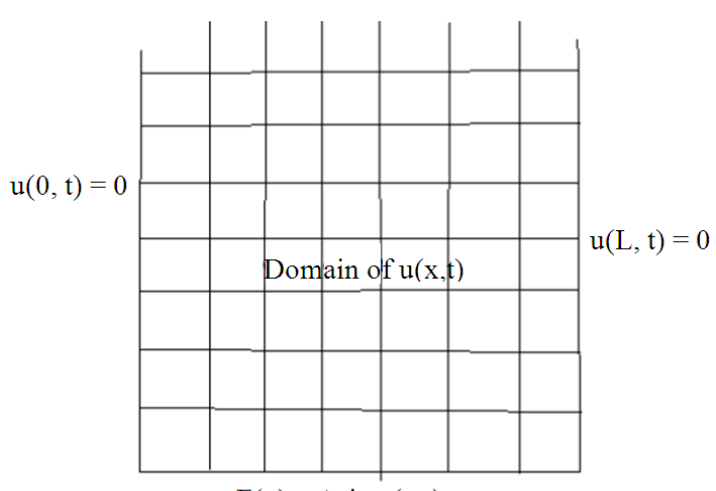

$\mathrm{F}(\mathrm{x})=\mathrm{A} \operatorname{sian}(\pi \mathrm{x})$

(b)

Fig. 2. (a) sketch showing a plucked string (b) grid showing the problem domain of $u(x, t)$

Thus:

$\frac{\partial^{2} u}{\partial x^{2}}=\frac{\rho \partial^{2} u}{T \partial t^{2}}$

Introducing the constant $c^{2}=\frac{T}{\rho}$, Equation becomes:

$\frac{\partial^{2} \mathrm{u}}{\partial \mathrm{x}^{2}}=\frac{1}{\mathrm{c}^{2}} \frac{\partial^{2} \mathrm{u}}{\partial \mathrm{t}^{2}}$

Equation (1.7) is the classical wave equation in oneDimension (1D), $\frac{\partial^{2} u}{\partial x^{2}}$ represents the 2nd partial derivative of the wave function with respect to the displacement;,$\frac{\partial^{2} \mathrm{u}}{\partial \mathrm{t}^{2}}$ represents the 2 nd partial derivative of the wave function with respect to time; $t$. The constant c represents the wave speed and it is dependent on the tension in the string and linear density of the string.

\section{MATERIALS AND METHODS}

\subsection{Introduction}

Partial Differential Equations (PDEs) can be solved numerically using finite difference method, Jain (2003). We write MatLab program for finite difference method to generate numerical solution to one Dimensional (1D) wave equation. As Kermit (1992) explained "MatLab is an abbreviation for Matrix Laboratory". It is interactive matrix based software for scientific and engineering calculations, which can be used to solve complex numerical problems.

\subsection{Central Difference Methods}

The central difference scheme for Equation (1.7) is obtained using Tailor's series expansion, Jain (2003) and Alejandro (1994). Thus, we then have:

$$
\begin{aligned}
& \frac{\{u(x+\partial x, t)-2 u(x, t)+u(x-\partial x, t)\}}{(\partial x)^{2}}=\frac{1}{c^{2}} \\
& \frac{\{u(x, t+\partial t)-2 u(x, t)+u(x, t \partial t)\}}{(\partial t)^{2}}
\end{aligned}
$$

Introducing the $\mathrm{i}, \mathrm{j}$ notation such that $\mathrm{u}(\mathrm{x}, \mathrm{t})=\mathrm{u}_{\mathrm{i}, \mathrm{j}}$ and also assigning $\partial \mathrm{x}=\mathrm{h}$ and $\partial \mathrm{t}=\mathrm{k}$, Equation (2.1) becomes:

$\frac{u_{i+1, j}-2 u_{i, j}+u_{i-1, j}}{h^{2}}=\frac{1}{c^{2}} \frac{u_{i, j+1}-2 u_{i, j}+u_{i, j-1}}{k^{2}}$.

Rearranging Equation (2.2) and putting the wave number $\lambda=\frac{\mathrm{ck}}{\mathrm{h}}$ :

$$
u_{i, j+1}=\lambda^{2} u_{i-1, j}+2\left(1-\lambda^{2}\right) u_{i, j}+\lambda^{2} u_{i+1, j}-u_{i, j-1} .
$$

Since the string is fixed at both ends, we have the boundary conditions $\mathrm{u}(0, \mathrm{t})=\mathrm{u}(\mathrm{L}, \mathrm{t})=0$.

These conditions are satisfied by the function $\mathrm{f}(\mathrm{x})=$ $A \sin (\pi x)$ when $L=1$ and $A$ is a constant. Or $f(x)=x$ (L-x) for any length $\mathrm{L}$ of the string.

To start the finite difference scheme we need the value of $u_{i,-1}$ in order to calculate the value of the wave function at the first time step, (i.e., at, $\mathrm{i}=0$ and $\mathrm{j}=0$ ). Evans et al. (2000) suggests the use of the initial velocity of the string.

That is Equation (2.4): 


$$
\frac{\delta \mathrm{u}(\mathrm{x}, 0)}{\delta \mathrm{t}}=\mathrm{g}(\mathrm{x})
$$

In this case the string is plucked from rest, so the initial velocity $\mathrm{g}(\mathrm{x})=0$. Now from Tailor's series we have Equation (2.5):

$$
\frac{u(x, t+\partial t)-u(x, t-\partial t)}{2 \partial t}=g(x)
$$

In the $\mathrm{i}, \mathrm{j}$ notation we have Equation (2.6):

$$
\mathrm{u}_{\mathrm{i},-1}=\mathrm{u}_{\mathrm{i}, 1}-2 \partial \operatorname{tg}(\mathrm{x}) \text {. }
$$

When we substitute $\mathrm{u}_{\mathrm{i},-1}$ in Equation (2.3), we get Equation (2.7):

$$
u_{i, j+1}=\frac{1}{2} \lambda^{2} u_{i-1}(x)+\left(1-\lambda^{2}\right) u_{i, j}(x)+\frac{\lambda^{2}}{2} u_{i+1}(x)+\partial \operatorname{tg}(x)
$$

But $g(x)=0$ :

$$
\Rightarrow \mathrm{u}_{\mathrm{i}, \mathrm{j}+1}=\frac{1}{2} \lambda^{2} \mathrm{u}_{\mathrm{i}-1}(\mathrm{x})+\left(1-\lambda^{2}\right) \mathrm{u}_{\mathrm{i}, \mathrm{j}}(\mathrm{x})+\frac{\lambda^{2}}{2} \mathrm{u}_{\mathrm{i}+1}(\mathrm{x})
$$

Equation (2.8) is the finite difference scheme used in the analysis of the wave function $\mathrm{u}(\mathrm{x}, \mathrm{t})$ at different values of the string parameters.

\section{RESULTS}

\subsection{Introduction}

The solution to the wave Equation (1.7) varies greatly with changes in the string variables. Using Equation (2.8), with the sine function; $\mathrm{f}(\mathrm{x})=0.2 \mathrm{sin}$ (x) for the condition $(\mathrm{t}=0)$, we wrote MatLab m-file program for Equation (2.3) and used it to find the numerical solution to the wave function $\mathrm{u}(\mathrm{x}, \mathrm{t})$ at different values of the string parameters.

We analyzed the nature of the wave forms produced by a guitar string in terms of the string variables. Results showed that changes in the length of the string tension and linear density of the string as well as changes in the steps sizes $(\partial \mathrm{x}$ and $\partial \mathrm{t})$; change the nature of wave form. Changes in tensions and linear density are done by varying the wave constant $c$ while changes in the step sizes are done by varying $\lambda$.

For a guitar string we have $0 \leq x \leq L, t>0$ where $L$ is the length of the string and $t$ represents time, with initial and boundary conditions Equation (3.1 and 3.2):

$\mathrm{u}(\mathrm{x}, 0)=\mathrm{f}(\mathrm{x})=0.2 \sin (\mathrm{x})$

And:

$$
\frac{\delta \mathrm{u}(\mathrm{x}, 0)}{\delta \mathrm{t}}=0
$$

\subsection{Variation in Values of Length $l$, the wave Constant $c$ and Steps Sizes, $h$ and $k$}

The constant $c=\sqrt{\frac{T}{\rho}} ; T$ represents the tension in the string and $\rho$ linear density of the string. The value of $c$ will increase with lower values of $\rho$ and higher values of $\mathrm{T}$. Results for different values of $\mathrm{c}$ are shown in the Fig. 3-12. When $c$ is high the wave form is non uniform as seen in Fig. 6.

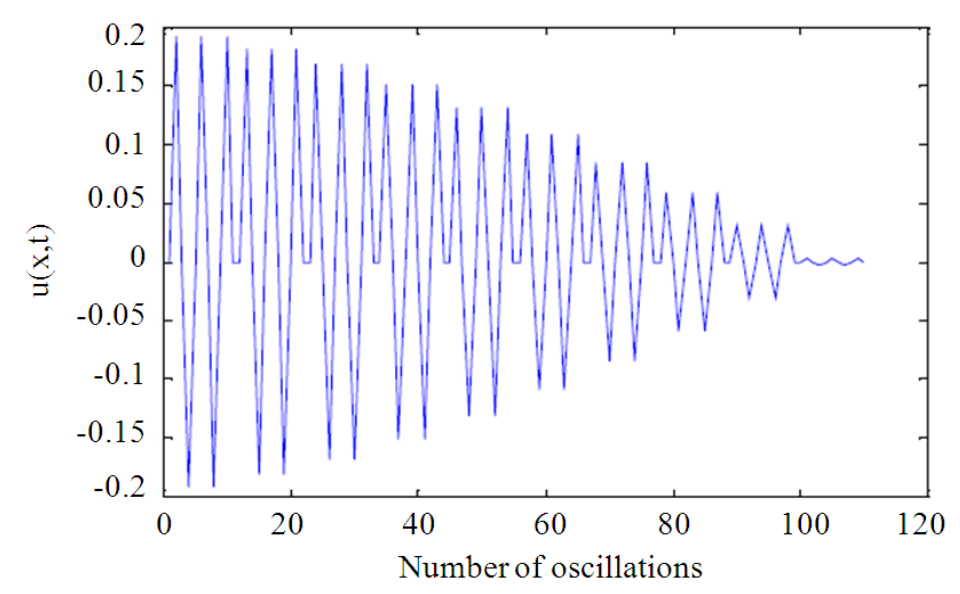

Fig. 3. Wave form, a plot of $\mathrm{u}(\mathrm{x}, \mathrm{t})$ at $\mathrm{L}=0.8, \mathrm{t}=0.5, \mathrm{c}=1$ and $\lambda=1$ 
Mango, J.M. et al. / Journal of Mathematics and Statistics 10 (2): 139-147, 2014

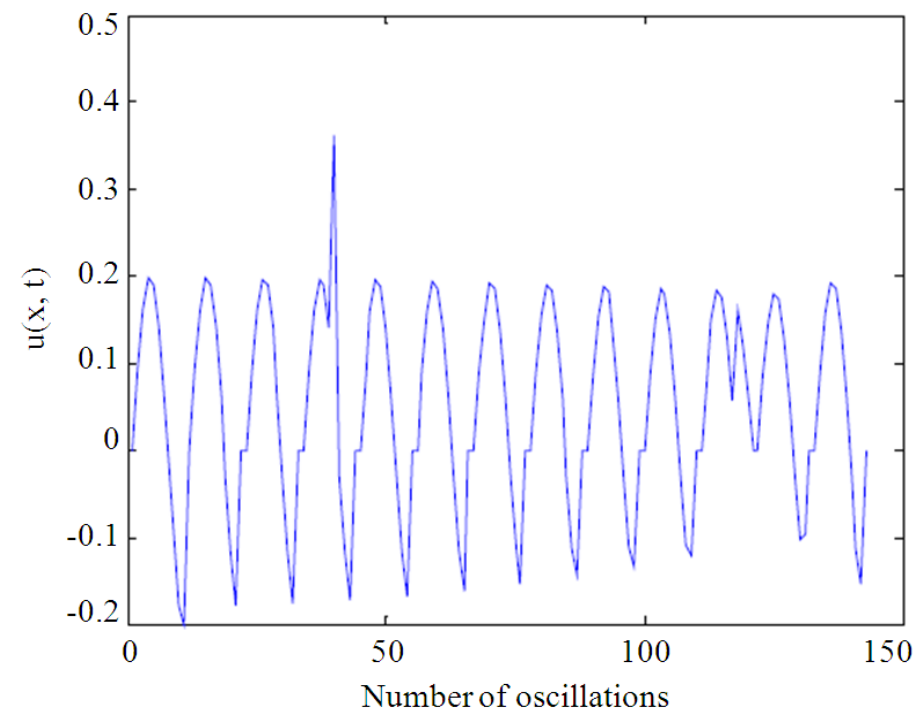

Fig. 4. A plot of $\mathrm{u}(\mathrm{x}, \mathrm{t})$ at $\mathrm{L}=1.5, \mathrm{t}=0.5, \mathrm{c}=2$ and $\lambda=1$

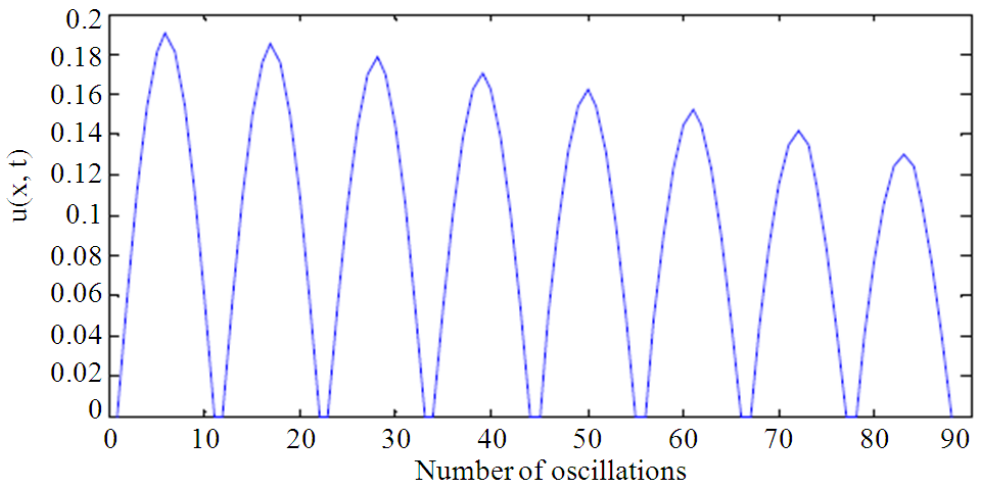

Fig. 5. Wave form, a plot of $u(x, t)$ at $L=1, t=0.5, c=2$ and $\lambda=4$

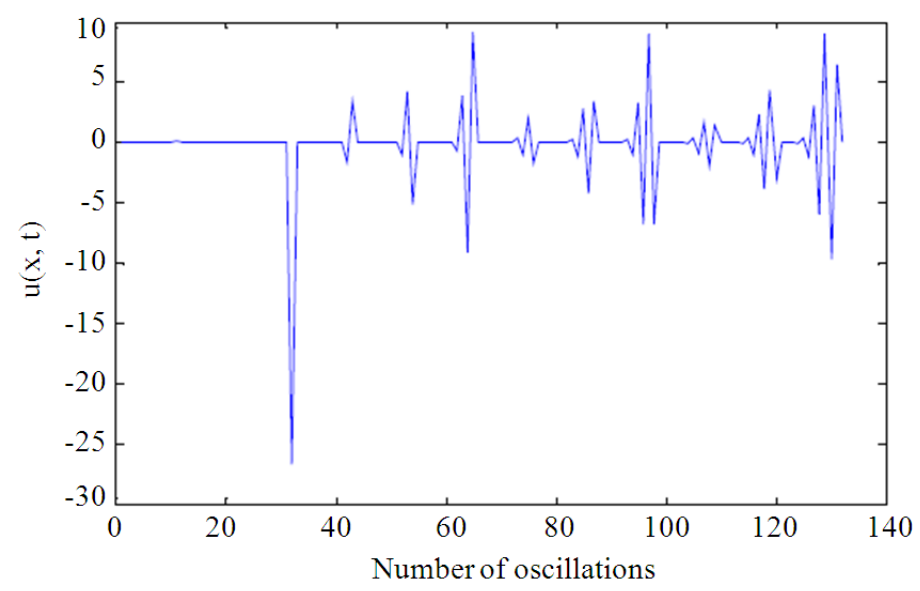

Fig. 6. Wave form, a plot of $\mathrm{u}(\mathrm{x}, \mathrm{t})$ at $\mathrm{L}=1, \mathrm{t}=1, \mathrm{c}=5$ and $\lambda=5$ 
Mango, J.M. et al. / Journal of Mathematics and Statistics 10 (2): 139-147, 2014

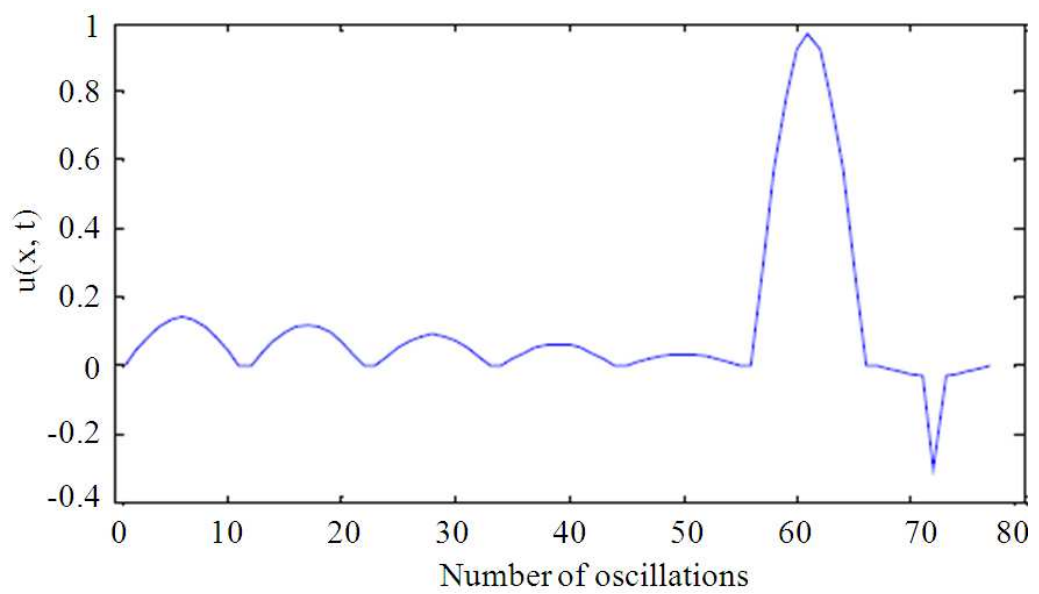

Fig. 7. Wave form, a plot of $\mathrm{u}(\mathrm{x}, \mathrm{t})$ at $\mathrm{L}=1, \mathrm{t}=0.5$ and $\mathrm{c}=2$ and $\lambda=2$

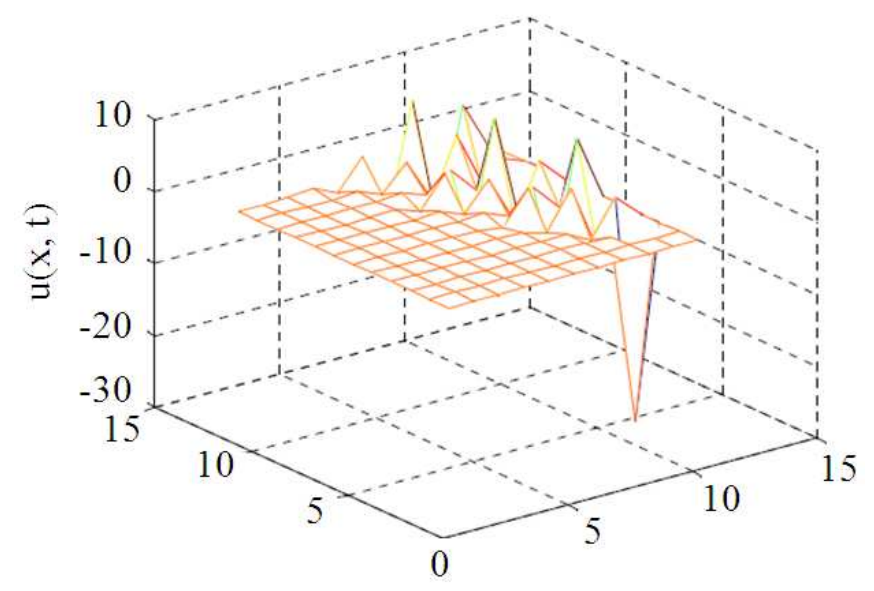

Fig. 8. 3D wire mesh plot of $u(x, t)$ at $L=1, t=1, c=3$ and $\lambda=3$

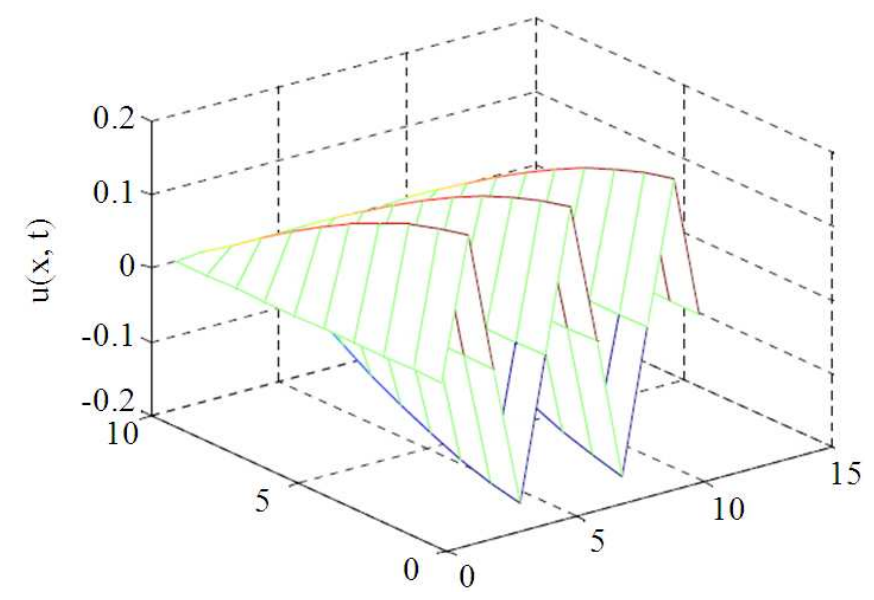

Fig. 9. 3D wire mesh plot of $u(x, t)$ at $L=0.8, t=0.5, c=1$ and $\lambda=1$ 
Mango, J.M. et al. / Journal of Mathematics and Statistics 10 (2): 139-147, 2014

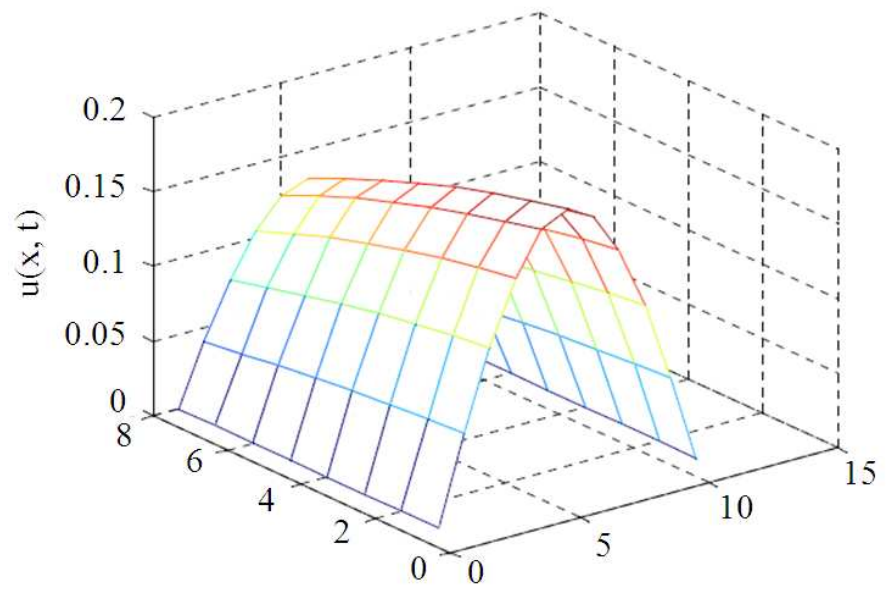

Fig. 10. 3D wire mesh plot of $u(x, t)$ at $L=0.5, t=0.5$ and $c=1$

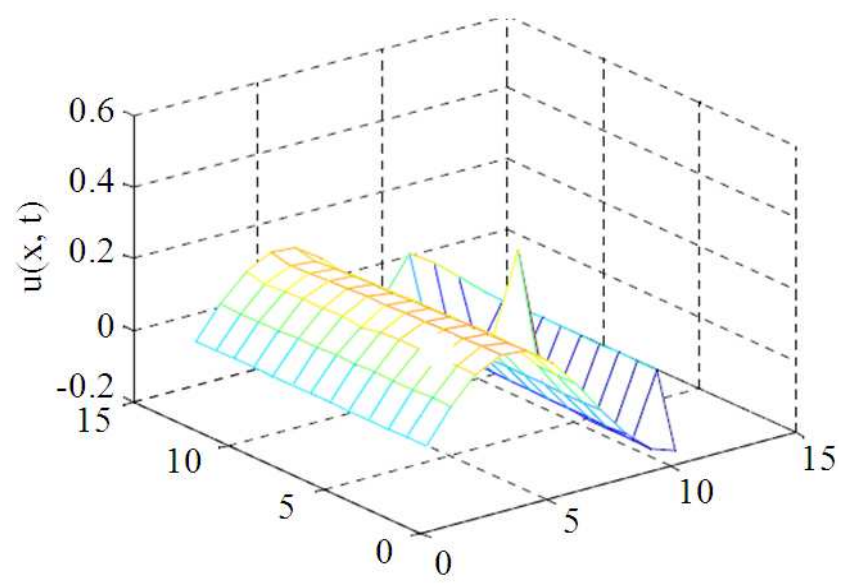

Fig. 11. 3D wire mesh plot of $u(x, t)$ at $L=1.5, t=0.5, c=2$ and $\lambda=1$

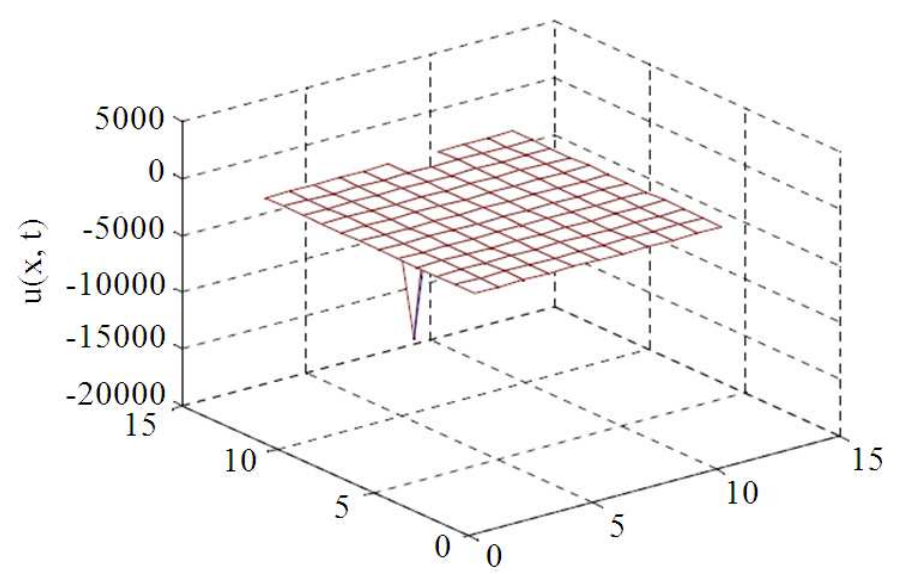

Fig. 12. 3D wire mesh plot of $u(x, t)$ at $L=1, t=1, c=1$ and $\lambda=4$ 
This means non uniform frequency of vibrations. Since c depends on tension $\mathrm{T}$, it means to have a mixture of waves with different amplitudes and frequencies, strings should be stretched to different tensions.

For shorter strings at lower values of $c(c \leq 2)$, we have many vibrations which die out faster; evident in Fig. 3-5. In Fig. 3, the amplitude falls just after $20^{\text {th }}$ mark (see horizontal axis) whereas in Fig. 4, the amplitude is nearly uniform up to the $150^{\text {th }}$ mark. As Mark (2004) stated "The amplitude of the wave is a measure of the pressure of its oscillations and corresponds to the volume of the sound". The variation in amplitudes due to differences in the length of the strings makes a guitar string to produce varied volumes. The guitar has 'infinitely many strings', because every time a string is tapped against the plate the effective length that is free to vibrate changes. Varied length means varied amplitude and pitch. The varied volumes are organized by the guitar player to produce music.

The effect of the step sizes; $h$ and $k$ is seen by varying $\lambda$, since $\lambda=\frac{\mathrm{ck}}{\mathrm{h}}$. Higher values of $\lambda$ produce wave forms with non uniform amplitude and frequencies. This means accuracy is lost at higher values of the step width. Compare Fig. 3, $\lambda=1$, Fig. 6, $\lambda=5$ and Fig. 7, $\lambda=2$.

The value $\lambda$ also depends on the wave velocity $\mathrm{c}$, since $\left(\lambda=\frac{\mathrm{ck}}{\mathrm{h}}\right)$, but $\mathrm{c}$ is also dependent on the initial force with which the string is plucked, strummed or struck. In this sturdy, it was not possible to mathematically analyze the effects of different values of the initial force. However, it seems clear that while playing the guitar, the strings just need appropriate force to make them vibrate with stable frequencies.

\subsection{The Wave Forms for Different Values of String Variables}

In the plot of Fig. 3 where, $\mathrm{L}=0.8$, the wave form (vibrations) die off faster, around the $100^{\text {th }}$ mark, compared to the one in Fig. 4 where $\mathrm{L}=1.5$

The plot Fig. 4 shows a wave form with a stable and fairly uniform frequency. The parameters here are nearly average. However the sharp peak with 'amplitude', $>0.2$ and also after the $100^{\text {th }}$ mark, the wave oscillated above the rest line. This anormally could have occurred due to truncation errors incurred in deriving the finite difference scheme or due to higher value of $c .(c=2)$.
The key thing to note in plot of Fig. 5 is that the wave existed only above the rest position, which may not be true. This could have been due to the step sizes, $\lambda=4$. The estimated solution is not accurate.

The plot of Fig. 6 shows the effect of the high vales of $\lambda$. At $\lambda=5$, the frequency is not uniform also extreme and non uniform amplitudes are observed. This means the computed values of $\mathrm{u}(\mathrm{x}, \mathrm{t})$ are not any near in agreement with exact values.

In the plot Fig. 7 the string did not vibrate for long, because $c=2$ is relatively high. The vibrations died to zero just before the $80^{\text {th }}$ mark (see horizontal axis). Also some extreme vales are observed far from the theoretically expected pattern. When compared with Fig. 5, much the oscillations in Fig. 5 continued passed the $80^{\text {th }}$ mark when $\lambda=4$, the solution seem out of trend of a wave, it is only above the rest position.

\subsection{The Three Dimensional Plot of $U(x, t)$, Figure 8-12}

At high values of $\lambda$, Fig. 8 the approximations are really poor. For a normal wave, this would show up and own folding plane as it nearly appears so in Fig. 11, where $\lambda=1$.

Note that Fig. 9 is a 3D plot of the waveform of Fig. 3. It is representative of the wave nature.

In the plot of Fig. 10, the vibrations (oscillations) appears to have been noticeable for just one cycle and die off. It could be due to small value of $L$, shortest in among all the sets of data.

The plot of Fig. 11 shows a better approximation to $\mathrm{u}(\mathrm{x}, \mathrm{t})$. Undulating plane, hill and slopes like it is on earth surface. Note. The data used to plot this is the same as that use in Fig. 4.

The set of result Fig. 12 also gives a poor approximation to the wave function. The plot shows no part of the wave above the rest position, $u(x, t)=0$. The value $\lambda$ at 4 may be too high for accurate results.

\section{DISCUSSION}

Following from the analysis of the different wave forms and mesh plot of the wave function, we can make some discussions about the wave from the a guitar string.

\subsection{The Length of the String}

The frequency of vibration of a guitar sound (pitch) varies greatly with the free length of the string. When the free length is short, the vibration is faster and shorter and this produces a high pitch. But guitars have strings of the same length; however, the process 
of playing involves tapping the string against the finger board. This process makes it possible for variation in the free length of the string, hence producing a mix of harmonics.

\subsection{The Value of $C$}

Since the wave velocity $c=\sqrt{\frac{T}{\rho}}$ where $T$ is the tension in the string and $\rho$ is its linear density, it means variation in $c$ effectively means variation in the tension and density. From the different plots in Fig. 4-12, increasing $\mathrm{T}$ means an increase in $\mathrm{c}$ which is the wave speed. Thus, to have superposition of different waves, it is important to have string stretched to different tension. Related to that, it may be advisable to have strings of different linear densities. This may mean to have string made of different materials or the same material but different cross sectional area.

\subsection{Other Parts of the Guitar-The Sound Board}

The sound board is one other significant part of a classical guitar. It serves to transmit the vibration of the string to the vibration of the surrounding air, which creates pressure differences in the immediate surrounding which are detected as sound. Some materials make better sound boards. As John (1999) wrote "A guitar with a graphite sound board has a unique sound as does a guitar with a spruce soundboard, or a cedar sound board, or a sound board made out of old garbage can". Some materials when used as sound board have high damping effects.

\section{ACKNOWLEDGEMENT}

The researchers are grateful for the financial support given by the International Science Program (ISP) at Uppsala University in Sweden. The support extended was used in the preparation and publication of this article.

\section{REFERENCES}

Alan, G., 2000. Mechanical waves. Cornel University. Alejandro, L.G., 1994. Numerical Methods for Physics. 1st Edn., Prentice Hall, Englewood Cliffs, ISBN-10: 0131519867. pp: 368.

Almazroi, A.A., 2011. A fast hybrid algorithm approach for the exact string matching problem via berry ravindran and alpha skip search algorithms. J. Comput. Sci., 7: 644-650. DOI: 10.3844/jcssp.2011.644.650

Al-mazroi, A.A. and N.A. Rashid, 2011. A fast hybrid algorithm for the exact string matching problem. Am. J. Eng. Applied Sci., 4: 102-107. DOI: 10.3844/ajeassp.2011.102.107

Evans, G., L.J. Black and P. Yardley, 2000. Numerical Methods for Partial Differential Equations. 1st Edn., Springer, Berlin, ISBN-10: 354076125X. pp: 290.

Jain, M.K., 2003. Numerical Methods for Scientific and Engineering Computation. 1st Edn., New Age International, ISBN-10: 8122414613. pp: 844

John, A.D., 1999. Rainsong graphite guitars.

Kermit, S., 1992. MatLab Premier. 2nd End., Prime Park Way, Natrick.

Lutz, T. and R. Rudolf, 2000. Multidimensional String Models. Proceedings of the Online Symposium for Electronic Engineers (SEE' 00).

Mark, R.P., 2004. Musical synthesis in matlab. College Math. J., 35: 396-399.

Ron, K., 2002. General wave motion-school for champions. 